\title{
RIFLESSIONI SULLA TRADUZIONE nell'Ottocento Messicano
}

\author{
Fernando Ibarra CháveZ*
}

ABSTRACT: Per tutto il secolo XIX, la traduzione di letteratura italiana nel Messico fu un'attività praticata soprattutto dai poeti. Come mestiere artistico e professionale, alcuni membri dell'élite intellettuale del paese videro la traduzione come un problema letterario e il traduttore fu valutato come un professionista specializzato. Le prime riflessioni sul tema le troviamo nella rivista Miscelánea di José María Heredia e, col passare del tempo, vedremo che i ragionamenti intorno alla traduzione cambiarono, al punto che alla fine del secolo Francisco Sosa pubblicò un commento analitico a proposito di tre traduzioni (spagnole e messicane) della Gerusalemme Liberata del Tasso.

PAROLE CHIAVE: traduzione; Messico; Ottocento; Heredia; Sosa.

RESUMO: Por todo o século XIX, a tradução de literatura italiana no México foi uma atividade praticada principalmente por poetas. Como prática artística e profissional, alguns membros da elite intelectual do país viram a tradução como um problema literário e o tradutor como um profissional especializado. As primeiras reflexões sobre o tema estão na revista Miscelánea de José María Heredia e, com o passar do tempo, veremos que as opiniões sobre a tradução mudaram, até Francisco Sosa publicar, no final do século, um comentário analítico a propósito de três traduções (espanholas e mexicanas) da Gerusalemme Liberata [Jerusalém Libertada], de Tasso.

PALAVRAS-CHAVE: tradução; México; século XIX; Heredia; Sosa.

* Universidad Nacional Autónoma de México, Cidade do México (México) fibarramx@yahoo.it Processo n.2013/20971-0 Fundação de Amparo à Pesquisa do Estado de São Paulo (FAPESP)

DOI: http://dx.doi.org/10.11606/issn.2238-8281.v0i34p103-111 
ABSTRACT: Throughout the nineteenth century, translating Italian literature in Mexico was an activity practiced specially by poets. This artistic and professional practice inspired some members of the intellectual elite of the country interested in translation as a literary problem and in translators as specialized professionals. First reflections about translation were published in the journal Miscelánea, directed by José Maria Heredia. Throughout the century, we will see how some perceptions of translation changed, until the publication of a professional commentary by Francisco Sosa about three translations (Spanish and Mexican) of the Tasso's Gerusalemme Liberata.

KEYWORDS: translation; Mexico; XIXth century; Heredia, Sosa. 
secolo XIX è, senza dubbio, uno dei periodi più turbolenti della storia moderna del Messico. È il secolo dell'indipendenza, delle invasioni straniere, dello sviluppo del nazionalismo e del debutto del paese sullo scenario internazionale della modernità industriale. L'effervescenza si manifesta anche nel campo delle arti e della letteratura: l'Ottocento messicano è il momento delle critiche all'oppressione spagnola, dell'adozione dello stile neoclassico, dell'arrivo del Romanticismo e del consolidamento di una letteratura con pretese di originalità e con una costante ricerca identitaria e nazionalistica, che si conclude con l'arrivo del positivismo.

L'eterogeneità dell'Ottocento, però, non era del tutto incline a imporre modelli a lungo termine. Se all'inizio del secolo gli autori latini avevano offerto terreno fertile per l'esercitazione mimetica, il romanticismo scosse le certezze di una volta e, quindi, le imitazioni ampliarono il panorama per prendere come spunto autori meno noti. I classici si trovarono sempre fra i modelli più meritevoli. Dato che molti autori ritenevano che le lingue romanze fossero in evidente svantaggio qualitativo di fronte al latino, il fatto di tradurre dal francese o dall'italiano poteva essere interpretato come un'audacia perché, oltre a non perpetuare i principi dell'arte classica, le traduzioni di nuovi autori permettevano la diffusione di idee all'interno di una tradizione classicheggiante ormai consolidata, generando così una crisi di valori estetici e concettuali i quali, tuttavia, offrirono motivi di riflessione lungo le prime decadi del secolo.

In quest'atmosfera, la traduzione in Messico non fu solo una pratica editoriale, bensì uno 
strumento pedagogico per coloro che avevano l'intenzione di entrare nel mondo della poesia. I poeti messicani vedevano nella traduzione un esercizio di formazione professionale simile a quello praticato dai pittori delle accademie che riproducevano sculture classiche o dipinti rinascimentali. Anche per loro la copia contribuiva a far capire in modo pratico i meccanismi tecnici dell'arte che desideravano emulare.

Le opinioni sull'attività di tradurre nell'Ottocento generalmente apparvero pubblicate in riviste culturali e negli studi introduttori che accompagnavano i libri di testi tradotti, quasi sempre per sottolineare il valore della traduzione proposta. Grazie a queste riflessioni, oggi abbiamo informazioni importanti che ci permettono di capire quali erano le difficoltà che affrontavano i traduttori, come si giustificava e si valutava il loro lavoro, quali connotazioni aveva la traduzione per il classicismo, il romanticismo, il nazionalismo e altre tendenze ideologiche. Inoltre si può individuare l'approccio dei commenti secondo il tipo di pubblicazione in cui circolavano. Infatti non si giudicava con gli stessi criteri una poesia tradotta in una rivista letteraria e un testo scientifico in un libro. Nel caso messicano, al posto di una produzione saggistica specializzata, troviamo delle piccole critiche o commenti generali nei confronti dell'opera di un autore, in cui si mette in luce il processo di traduzione, a volte in modo descrittivo, a volte con un forte senso critico. Sono ugualmente importanti sia il giudizio negativo sia quello encomiastico visto che, a causa della scarsità di testi sistematici sul problema della traduzione, l'accumulazione delle notizie giornalistiche forma nell'insieme un complesso "manuale", che offre notizie su meccanismi, risultati, conseguenze e ricezione critica della traduzione.

La traduzione di prosa di solito non meritò la stessa critica che la traduzione di versi. Vista come attività accessoria, la pratica della traduzione di prosa era incombenza di persone meno esperte nell'arte letteraria: infatti troviamo spesso racconti e saggi - originariamente scritti in lingue moderne - tradotti da donne. La traduzione faceva parte del curriculum di molti intellettuali, anche se non erano poeti. Frequentemente, quando si parla di personaggi centrali della vita culturale o politica, si menzionano le loro traduzioni, come parte del rito di passaggio di qualsiasi aspirante all'élite intellettuale.

Nella stampa del Messico indipendente le traduzioni erano comunissime. Per citare un caso emblematico, la rivista Miscelánea - fondata dal poeta cubano José María Heredia nel 1829 - pubblicò per la prima volta alcuni testi di autori classici, ma anche testi di Goethe, Byron e, naturalmente, di autori italiani. Miscelánea ebbe la missione di diffondere le novità letterarie del mondo e di offrire riflessioni critiche sull'opera di autori messicani e stranieri (ossia francesi). 
Con queste aspirazioni, la traduzione serviva come veicolo per la divulgazione di testi di cui non esisteva una versione in spagnolo. Sebbene nella rivista non si trovi un articolo sulla traduzione, nelle sue pagine ci sono dei commenti su quello che è o dovrebbe essere un buon traduttore: così possiamo ricostruire i principi basilari che costituivano la ricezione della traduzione secondo Heredia. In primo luogo, si deve chiarire che Heredia sostenne sempre che le lingue moderne erano imperfette, pertanto non avrebbero mai potuto riflettere la bellezza della lingua latina. Heredia considera che in ogni lingua ci sono tratti caratteristici inerenti, così essenziali ad essa che non vengono meno neanche quando il testo viene tradotto: se un gruppo di poesie di varie origini geografiche fosse tradotto nella stessa lingua, i prodotti finali permetterebbero comunque di raggrupparle secondo la loro origine. Oggi sarebbe difficile sostenere l'ipotesi della persistenza di tratti essenziali della lingua originale nel testo tradotto, ma nell'Ottocento l'affermazione sembrava valida e indiscutibile.

Una grande parte dei commenti pubblicati in Miscelánea si concentra, soprattutto, nella definizione del mestiere del traduttore, a partire dagli errori trovati nelle traduzioni. Con tono precettivo e sentenzioso, Heredia indica che "un traduttore deve solo impegnarsi nel ritrarre con fedeltà l'originale su cui lavora, con tutti i suoi difetti e bellezze, ma non dovrebbe emendare quello che non gli appartiene" (HEREDIA, 1829, p. 33). ${ }^{1}$ Sicuramente l'idea circolava già da tempo nel mondo, ma non dobbiamo dare per scontato che fosse una regola seguita da tutti, perché frequentemente ci troviamo di fronte a testi molto alterati dal traduttore, i cui interventi vengono giustificati con argomenti letterari. In molti testi sulla traduzione troviamo delle raccomandazioni del genere, discutibili, sì, ma frutto della consapevolezza che la traduzione era un' attività molto delicata, perché facilmente si poteva cadere in errori irrimediabili, ottenendo come risultato una traduzione non riuscita e, quindi, falsa. Dice Heredia: "Il traduttore che copia servilmente forme straniere, traveste la propria lingua e il testo originale, che non viene tradotto, ma calunniato. Il pittore che vuole fare un ritratto simile, prenda la fisionomia: colui che cerchi di esprimere fedelmente un classico, e mantenere tutti i suoi pensieri, provi a scrivere come lui avrebbe scritto in quella lingua, perché non si dovrebbero tradurre le parole senza il loro spirito"

1 "Un traductor sólo debe esforzarse a retratar fielmente el original que lo ocupa, con todos sus defectos y bellezas, pues no le toca enmendar lo que no le pertenece." 
(HEREDIA, 1830, p. 8). ${ }^{2}$

Per continuare la discussione, il poeta Manuel Carpio pubblicò una serie di considerazioni nell' articolo "Observaciones sobre la traducción de los poetas" (CARPIO, 1998, pp. 581-587). In questo saggio del 1830, l'autore elenca cosa si esige generalmente dal traduttore di poesia e, di conseguenza, cosa gli si critica più frequentemente. Il traduttore è obbligato a conservare a tutti i costi le caratteristiche dell'originale, vale a dire la versificazione, le rime e persino "il genio della lingua". Altri critici, forse più consapevoli della difficoltà o addirittura impossibilità di mantenere gli schemi metrici e ritmici, prescrivono che la traduzione rispetti solo le idee, le parole e lo stile. Per Carpio, il traduttore deve agire come il pittore di ritratti - ancora la comparazione - copiando in dettaglio ogni elemento del modello, senza aggiungere o togliere nulla, neppure quando la modifica potrebbe aiutare a migliorare l'aspetto del testo tradotto. Anche se l'intervento del traduttore potrebbe impreziosire il testo originale, è anche vero che rischia di aggiungere degli errori, che poi vengono attribuiti all'autore originale.

Una tendenza generale che Carpio riscontra nei traduttori è proprio il desiderio di abbellire i testi originali con aggiunte o soppressioni o, peggio ancora, parafrasando interi passaggi di testo al posto di tradurli. Per offrire un esempio di questo procedimento, nello stesso articolo Carpio presenta un frammento dell'Odissea tradotta da Chateaubriand e Bitabué. Due diverse traduzioni, sia per l'estensione che per la redazione: frasi in più, parole in meno, aggettivi presenti o assenti, chiose che non esistono nell'originale e parecchie omissioni. Dopo di che Carpio propone una traduzione della seconda ode del primo libro di Orazio, con la finalità di dimostrare che si può tradurre "senza cambiare nulla".

Tradurre autori latini era considerato come un utile esercizio di stile per i poeti messicani della metà del XIX secolo. Successivamente cominciò l'epoca degli autori romantici, più o meno contemporanei. È interessante notare che nell'elenco degli autori italiani tradotti in questo periodo non sono presenti né Petrarca né Boccaccio. Il primo forse perché era il fondamento della poesia amorosa del Barocco, poco amata o rifiutata tout court per i suoi legami con la poesia spagnola; il secondo perché aveva scritto in prosa la sua opera più importante, e c'erano già delle buone traduzioni del Decameron in francese. Perciò sorprende che Carpio abbia

2 "El traductor que copia servilmente formas extranjeras, disfraza su idioma y su original, a quien no traduce, sino calumnia. El pintor que quiere hacer un retrato parecido, toma la fisionomía: el que pretenda expresar fielmente a un clásico, y conservar todos sus pensamientos, procure escribir como él habría escrito en aquella lengua, porque no deben traducirse las palabras, sin el genio." 
incluso, nell'edizione delle sue poesie, due composizioni di Pietro Metastasio (1698-1782): "La Libertà" e "Palinodía", testi indirizzati all'ex-amata Nice, dove il poeta italiano fa una satira del petrarchismo. Cito la prima strofa:

\begin{tabular}{|l|l|}
\hline Grazie agl'inganni tuoi, & Gracias a tantas perfidias \\
al fin respiro, o Nice, & Al fin ya respiro, Nice: \\
al fin d'un infelice & Al fin, de aqueste infelice \\
ebber gli Dei pietà: & El cielo tuvo piedad. \\
sento da' lacci suoi, & Suelta el alma de prisiones \\
sento che l'alma è sciolta; & De mí me conozco dueño, \\
non sogno questa volta, & Y al presente ya no sueño, \\
non sogno libertà. & No sueño en la libertad. \\
\hline
\end{tabular}

Per quanto riguarda gli aspetti formali, le licenze poetiche del traduttore non sono violente. I settenari di Metastasio si trasformano in ottonari, mentre lo schema ritmico abbc addc cambia in modo sottile in abbc deec, rispettando il verso acuto della rima c. Il presunto rispetto per l'ordine delle idee nei versi è anche raggiunto: tuttavia è strano l'uso dell'aggettivo determinativo 'aqueste' -arcaismo- e, ovviamente, l'e' epentetica dell'aggettivo 'infelice'.

Molto interessante risulta "De las traducciones", un piccolo articolo apparso in El Diario de los Niños in cui si spiegano le caratteristiche di una buona traduzione. L'autore - forse un editore e non un professionista delle belle lettere - sostiene che l'attività traduttiva, anche se molti la esercitano con assoluta serietà, di solito è funestata da vizi nocivi nati dalla mancanza di esperienza, dall'ignoranza o dalla capricciosa libertà del traduttore. Per conservare la bellezza dell'originale, il traduttore non deve assoggettarsi alla letteralità del testo né prendersi la libertà di manipolarlo a suo agio. Idealmente, l'autore suggerisce che il traduttore riesca ad appropriarsi dell'originalità del testo, come se fosse stato concepito da lui. In questo senso, la migliore traduzione sarebbe quella che non sembra una traduzione, ma una seconda creazione. Infatti, si afferma che il traduttore di un grande autore dovrebbe essere a sua volta un grande scrittore: solo così si può garantire la qualità del testo tradotto. Tutto parte da una fusione: "un traduttore non deve sviluppare le proprie idee con la stessa veste che indossava l'originale, ma deve seguirne il pensiero; non deve copiare le nude espressioni dello scrittore, ma scendere nel profondo dei suoi 
sentimenti, prendere tutte le passioni della sua anima, tutta la creatività della sua immaginazione, tutte le qualità del suo stile, e trasferire il genio dell'autore piuttosto che quello della lingua". (De las traducciones, p. 10) ${ }^{3}$ Si tratta di una visione notevolmente diversa da quella di Heredia o Carpio, che puntavano sempre sulla forma.

Quasi contemporaneamente José Joaquín Pesado pubblicò in El Año Nuevo del 1840 i primi tre canti dell'Inferno, "Fragmentos de la Divina comedia del Dante", in prosa (PESADO, 1840, pp. 32-43). Questa traduzione apportò nuova fama all'ormai celebre figura di Dante nel Messico. La traduzione non meritò molti commenti: sicuramente, come si è detto, perché il traduttore rinunciò alla versificazione e, quindi, si considerava che non c'era niente da criticare. La proposta è molto interessante perché, di fatto, vengono più o meno conservati gli elementi linguistici del fiorentino, senza aggiunte o omissioni evidenti, ma con certe imprecisioni nell'interpretazione del poema dantesco. Verso il 1860, Pesado tradusse frammenti di un altro testo italiano, $\mathrm{La}$ Gerusalemme Liberata di Torquato Tasso. Qui, l'esperienza del poeta messicano si rispecchia in ogni ottava tradotta. Sembrerebbe che prima di farla, Pesado prese in considerazione tutte le critiche alle traduzioni precedenti, per conservarne la forma, il contenuto, l'ideologia e, ovvio, gli elementi linguistici, senza prendersi delle licenze insolite in spagnolo.

Così, in mezzo all'eclettismo ottocentesco, arrivò il positivismo con una maniera diversa di vedere la traduzione. Nel 1885, Francisco Sosa scrisse Versiones castellanas de la Jerusalem libertada de Torcuato Tasso, un testo di critica alla traduzione molto interessante. Il critico analizza alcune traduzioni della Gerusalemme liberata. Per poter rendere "scientifico" il suo lavoro, presenta il testo di Tasso e una traduzione letterale in prosa, affinché il lettore possa essere sicuro di aver capito le parole italiane. Poi vengono presentate le versioni in spagnolo di José Joaquín Pesado, di José de la Pezuela Ceballos conde de Cheste e di Francisco Gómez Palacio. Non mi fermo qui a commentare l'analisi che ne risulta, ma credo importante sottolineare alcune riflessioni di Sosa. Per cominciare, l'autore indica che, sia in prosa che in versi, la traduzione deve mantenere il criterio di esattezza (exactitud) nell'interpretazione, cioè, "lo studio che segue l'ordine delle parole dell'originale; economia e distribuzione dei periodi, dividendoli come l'autore quanto lo consenta il senso del periodo e il genio della lingua in cui si traduce" (SOSA, 1885, p. 38).

3 "Un traductor no debe desenvolver sus ideas con el mismo vestido que tenía el original, sino seguir el pensamiento; no debe copiar las expresiones desnudas del escritor, sino descender a toda profundidad de sus sentimientos, tomar todas las pasiones de su alma, toda la actividad de su imaginación, todas las cualidades de su estilo, y trasladar el genio del autor más bien que el de la lengua." 
Per quanto riguarda la traduzione di versi, necessariamente le difficoltà aumentano. Una certa educazione letteraria e un tocco di genio poetico sarebbero necessari per chi volesse tradurre un autore di rilievo. Francisco Sosa, tuttavia, non ritiene che solo un poeta consumato sia qualificato per tradurre un altro poeta, anzi, è un rischio perché "il poeta di robusta ispirazione e di eccelsa produzione, abituato a dare volo libero alle sue idee, non riesce a gestire gli ostacoli che implicano le leggi da seguire da parte del traduttore. Il genio è creatore per eccellenz e, senza rendersene conto, mescola alle idee altrui le proprie idee; crede spesso che possa essere migliorata un'immagine, e la migliora; trova più poetica un'altra forma, e gliela dà" (SOSA, 1885 , p. 39). Sosa aggiunge che molti poeti di una certa qualità vedono la traduzione come un'attività secondaria e, di conseguenza, non le conferiscono il giusto valore. Quindi, chi sarebbe la persona che può apprezzare le virtù di un buon traduttore? Secondo Sosa gli uomini dotti, che conoscono bene ognuna delle difficoltà che un buon traduttore deve superare.

L'Ottocento messicano è ricco di riflessioni teoriche e metodologiche sulla traduzione. Sebbene per i poeti legati al neoclassico la traduzione fosse vista come un semplice esercizio letterario e il romanticismo la adoperasse strumentalmente come veicolo di diffusione, alla fine del secolo le considerazioni di Francisco Sosa dimostrano che quest'attività ancillare cominciò ad essere trattata con tutta la serietà che implica qualsiasi lavoro intellettuale e, di conseguenza, anche la figura del traduttore è stata rivalutata con nuovi parametri. C'è ancora molto lavoro da fare per capire con precisione i rapporti tra il traduttore, la teoria, la critica, le esigenze editoriali e le aspettative del pubblico.

\section{Riferimenti bibliografici}

CARPIO, M. Poesía [1860]. Ed. Fernando Tola de Habich. México: UNAM, 1998.

De las traducciones, El Diario de los Niños, Messico: 9-10, 2 gen.1840.

HEREDIA, J. M. Versos de J. Nicasio Gallego, recogidos y publicados por Domingo del Monte. Filadelfia: Imprenta del Mensajero: 1829. In Miscelánea, Messico: 1: 33, ott. 1829.

HEREDIA, J. M. Poetas franceses modernos. Jacobo Delille. In Miscelánea, Messico: 5: 8, gen. 1830.

PESADO, J. J., Fragmentos de la Divina comedia del Dante", in El Año Nuevo de 1840, Messico: 32-43, 1840.

SOSA, F. Versiones castellanas de la Jerusalem libertada de Torcuato Tasso. México: Secretaría de Fomento, 1885.

Recebido em 10/12/2016

Aprovado em 04/05/2017 